\title{
MaterialNature: An Opportunistic Paradigm of Architecture and Landscape Ecology
}

Marcus Farr

\begin{abstract}
In this space, small-scale coastal erosion, and natural growth become the vehicle for project based architectural speculation that leverages the resources of local landscapes and creates a new architectural trajectory based upon the need and aesthetic of ecology. Here, MaterialNature is a series of architectural entities that become landscapes over time, made from a mixture of residual materials designed specifically to be weatherized. Its design is intended to provide a potential architectural amenity in areas of recreational activity as well as areas devoid of human contact, while simultaneously realizing a potential for architecture to be born from waste or local material, and to return to nature without providing further waste. As such, this architecture moves from one of formal composition to one where the formal characteristics of local ecology and landscape become more apparent than the form itself.
\end{abstract}

Keywords: architectural ecology, erosion, landscape ecology, large-scale 3D printing, material innovation

The call for "Resilient Edges - Investigations, Strategies and Designs for the Ecological Resilience of Coastal and Delta Areas Around the World" presented a unique opportunity for those interested in the design and theory of the built environment to explore problems and solutions relative to the pressing problems of global environmental damage, climate change, materiality, along with a parallel potential for an architectural language of which permanence may not be the singular performative element. The subsequent content is situated at the intersection of water and land 
whereby the evidence of ecology, both manifest and specular, are at a point of considerable vulnerability. In this site, the variables of life are at once extremely noticeable and at times absent. This zone of "wrack and drift" is one where the physical artifact of architecture presents a need for a flexible, hybrid character, reflecting the needs of both the natural and the manmade, along with their respective infrastructure.

In the essay "Non-Anthropocentric Architectures," Michael Hensel responds to the issues of altered environmental conditions, "For several decades numerous politicians, biologists and architects have stated the need for a biological approach to some of the most pressing problems arising from the extensive impact of humankind on the natural environment." ${ }^{1}$ The 1987 report of the World Commission on Environment and Development, entitled Our Common Future and commonly known as the Brundtland Report, explored a broad range of sustainability concerns, including the fundamental necessity or preserving the abiotic (non-living) and biotic (living) environment and its associated processes:

Important are the vital life processes carried out by nature, including stabilization of climate, protection of watersheds and soil, preservation of nurseries and breeding grounds, and so on. Conserving these processes cannot be divorced from conserving the individual species within natural ecosystems. Managing the species and ecosystems together is clearly the most rational way to approach the problem. ${ }^{2}$

The "Non-Anthropocentric" essay continues with the question, "how are natural processes and ecosystems to be maintained? And what kind of disciplinary affiliation between architecture and biology is needed in order to tackle the complexity of the problems arising from the interaction between the human-made and the natural environment? To confront these issues it is necessary to focus all efforts on the question as to how the built environment can be 'in the service of' the natural environment." 3

As rising sea levels are projected to continue one meter before $2100,{ }^{4}$ there is cause for a specific dimensional area of speculation to be fertile ground for architecture, landscape architecture, ecological urbanism. At this point, it seems fitting that words such as barricade, barrier, block, dam, wall, facade, fence, screen, side, be replaced or reconsidered along side such words as accessible, susceptible, expanded, exposed, revealed, rolling, dehiscent, expansive, navigable, whereby the aesthetic of future intuitive programs can be embraced by fluctuating needs of both man and nature, and the performance of the drift zone be up-cycled to one of elevated performance. This need is made more important considering that many of the fastest growing cities in the world are along a coastline, and population is at a level higher than previously seen in our history. Even at the current state of global population, it is clear that our relationship to manmade 
and natural ecology is in need of radical cultivation and refinement on a multitude of scales.

Beaches have always been an ongoing setting for erosion and the effects of natural processes relative to materials and landscapes. In this setting natural selection can take its toll and ecologies are shaped because of what they are, where they are, and what they are made of, literally. In the twentieth century, this setting also, unfortunately, became prime territory for pollution, waste, and erosion due to manufacturing, lack of regulation and expanse of globalization whereby coastal shipping practices privilege transient international growth at the expense of local sustainability. In the special issue of Architectural Design titled "Cities of Dispersal," the essay "Our Beautiful Future," Martha Rosler describes "collaborative projects which were manifested at the 2003 Venice Biennale that joined together students and artists in imagining alternative publics to rescue the utopian hopes" ${ }^{5}$ of society. In these projects there is discussion of imaginary "post-places" whereby mankind perhaps would not recognize itself in the promised land of change and remediation. For the project MaterialNature, the imagined role of the "post-place" is a tidal zone where architecture is centered around a material agenda that gives way to landscape and a reconceptualization of the bionomic circuits of waste, erosion, ecology and opportunity.

In this space, influences of small-scale coastal erosion, and natural growth become the vehicle for project based architectural speculation that leverages localized material logics and creates a new architectural trajectory based upon the need and the aesthetic of ecology. In this state, MaterialNature is a series of architectural entities that become landscapes over time, made from a mixture of residual materials designed specifically to be weatherized. Its design is intended to provide a potential architectural amenity in areas of recreational activity as well as areas devoid of human contact, while simultaneously realizing a potential for architecture to be born from waste, and to return to nature without providing further waste. As such, this architecture moves from one of formal composition to one where the formal characteristics of local ecology and landscape become more apparent than the form itself.

Moving to a practical reading of the beach as a site, although non-scholarly, the manual How to Read a North Carolina Beach published (2004), discusses years of scientific observations taken by walking beaches and observing the materiality and found condition of the daily wrack zones caused by the ebb and flow of the tide. In this, the author states:

Much of the extraneous material on beaches is found in drift lines (wrack lines) which are the linear piles of natural and man-made objects that are left behind at the uppermost limit of the wave swash. The positions of the drift lines are a good indicator of the 
high tide or storm wave limit. On some beaches, especially the undisturbed ones, as many as three separate wrack lines can sometimes be seen. The lowest line of debris marks the normal high-tide line. The next line, a foot or two higher, is from the fullmoon tide, and the highest wrack line is from the last big storm. A walk into the dunes, into the center of the island, far from the shoreline, may reveal old lines left from significant flooding as long ago as ten of twenty years. ${ }^{6}$

The author's description of the found condition is one that illustrates a change in the material status of the site and indicates the natural history of day to day activity along this versatile but dimensionally thin landscape ecology. It tells of an ephemeral reading of the site based upon time, material, water, wind, gravity.

This reading is not always apparent. A variety of practices designed to supplement our day to day existence in modern society act to erase these natural readings. Actions such as beach grooming, sand modification, seaweed and shell harvest, erosion, illegal sand collection, coast-line fracking, urban runoff, sewage lines, improper drainage all act to modify or in some cases erase the natural state of being along the drift. Unfortunate in that the natural retention of this material, along with the ecology that accompanies it, can naturally work to maintain the delicate performative balance between landscape and ecology. The removal of material and the presence of introduced actions such as those stated above only work to create an exponential situation when added to the aforementioned

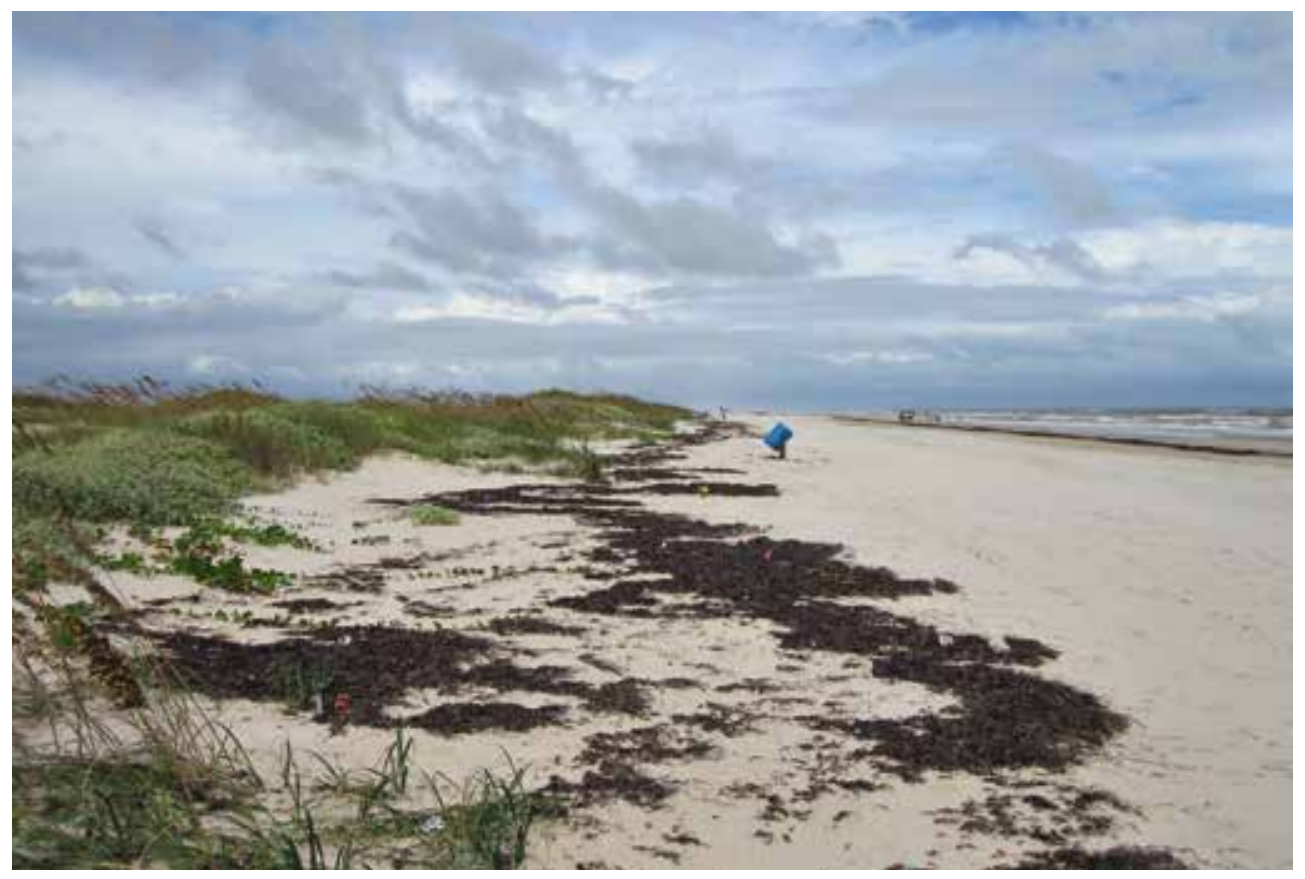

Figure 1. Drift lines and wrack zones. 
conditions of population growth and urbanization. The beaches are simply stripped bare, and what remains is a design question that begs for answers in a world where man controls the outcome.

Regardless of urbanization, beaches help support economies. Coastal communities depend on the income from tourists. In order to keep the beaches aesthetic, grooming typically occurs and is a widely used practice to keep the sand clear of both natural debris and trash, which is appealing to the public. It is obvious to scientists that this practice significantly disturbs the sandy beach ecosystem. Fortunately, in certain areas, the presence of pollution and irresponsible practices also bring with them the presence of sustainably minded people with a goal to manage current practices. However the task is monumental and requires input from scientists, government agencies, environmental groups, and concerned citizens to develop grooming practices that strike a better balance between the economic and ecological assets of the beach. The success of these modified beach maintenance practices demonstrates the importance of basing environmental management in sound science and supporting collaboration among many diverse stakeholders.

According to the 2017 scientific interview "Beach Ecology," 7 researchers view the high tide line as "an ecologically vibrant zone on the beach [...] grasses wash up to this zone, forming what is called the wrack line. As the wrack breaks down, it provides nutrients that form the base of the food chain on the beach, supporting everything from tiny invertebrates to shorebirds. Many nutrients from the wrack also filter through the sand and make their way back out to the water, supporting the near-shore marine

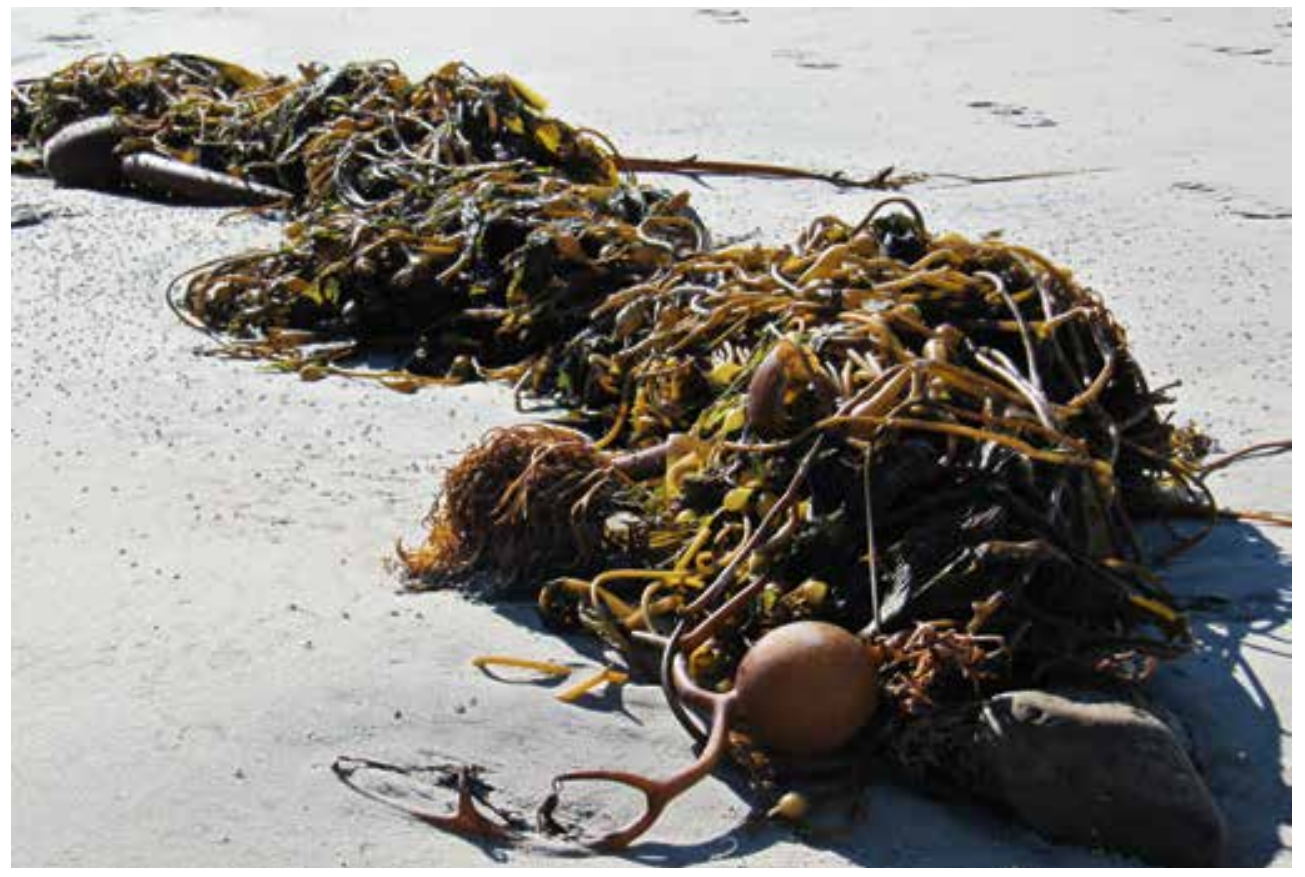

Figure 2. Beach wrack. 
ecosystem. ${ }^{8}$ Both wrack at the high tide line and plants growing on the upper beach help to reduce the impact of the wind, protecting the sand from erosion. These processes are essential to maintaining a healthy beach ecosystem." 9

\section{METHODOLOGY / PROCESS}

The altered environmental conditions of today can no longer be mastered with the architectural resources of the past. The relationship between biology and building is now in need of clarification due to real and practical exigencies.

The problem of environment has never before been such a threat to existence. In effect, it is a biological problem. ${ }^{10}$

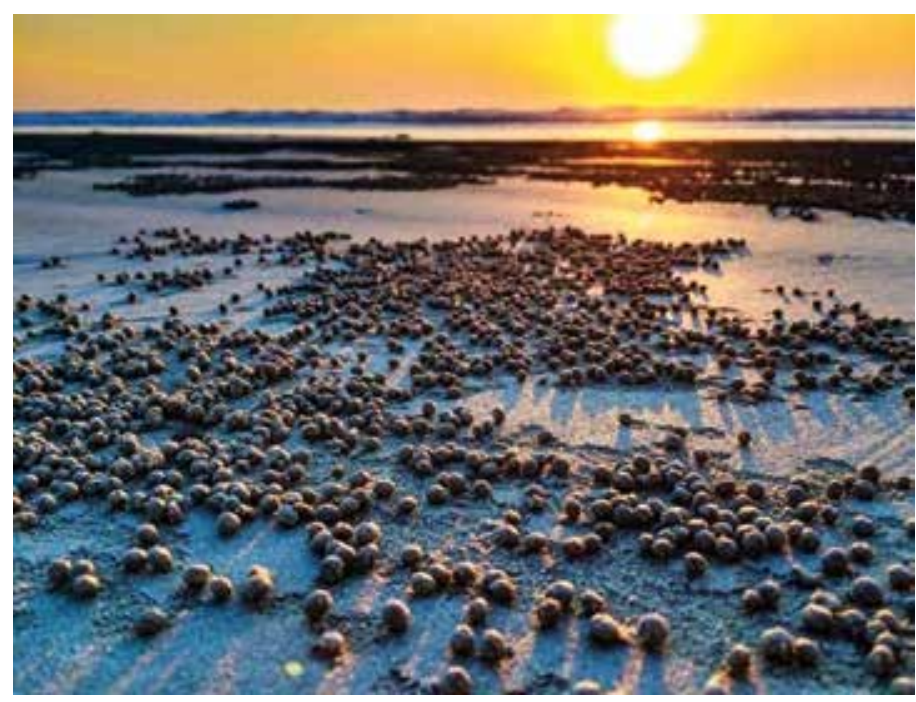

Figure 3. Bubbler crab deposits.

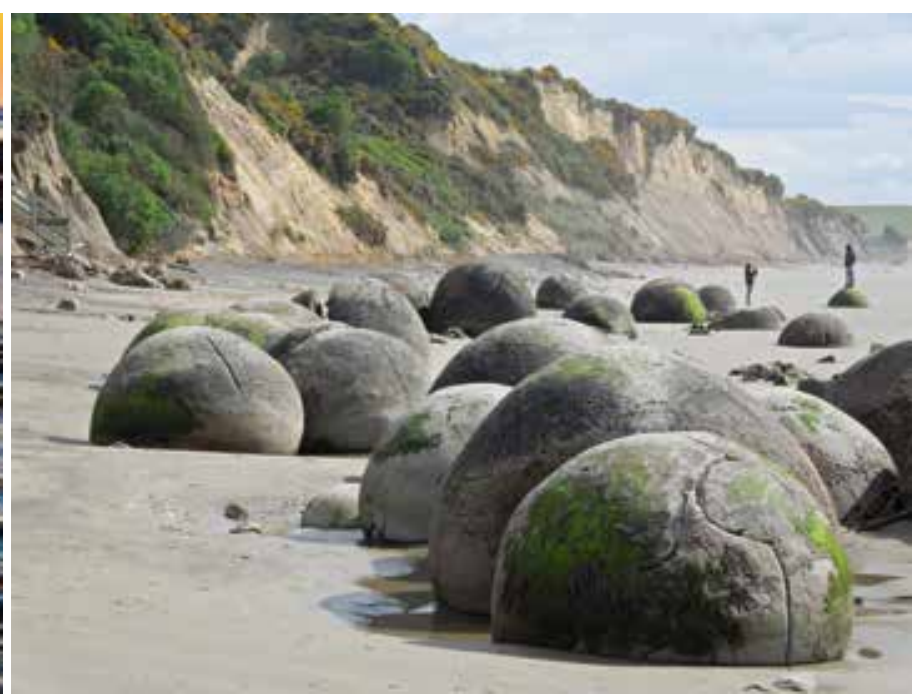

Figure 4. Moeraki boulders.

MaterialNature is not as interested in creating a built architectural object, but rather a series of architectural situations that involve mixtures of architectural material \& landscape ecology as ways to mesh the material gradients of the tidal zone, and ultimately formulate solutions to negotiate the collaborative relationships of site and erosion that make up the found conditions created by improper globalization, pollution and waste.

As a synthesizing of hybrid frameworks, the goal of the material palette is to demonstrate performative criteria needed for a healthy beach, and to subsequently generate experiences that explore local landscape phenomena such as flower gardens, corals, sand dunes and succulent groves. These plant pallets are designed according to the local ecology. The shape grammar of the architecture is inspired from localized conditions most appropriate to the stone formations found in the area and beneath 
the sand itself, such as those referenced by the geology of Moeraki Beach Boulders on New Zealand's Otago coast, or can even be inspired by smaller scale actions that lead to bigger propagations such as the activities of sand bubbler crabs, and then digitalized and compounded by effects of wind and salt water. The material palette is born organically via a step by step process that begins with large scale 3D printing with a Potter-bot in a university lab, then placed on site, covered with biological geotextiles on top of with local growing medium and subsequently seed-bombed and watered. Because it is important for the project to contribute as little as possible to the landfill and to the man-made footprint of each individual area, the material from the 3D-printers is the only purely non-native material involved.

This material is printed from a modified Potter-bot printer that normally prints large-scale pottery elements, but is in this case used to print with

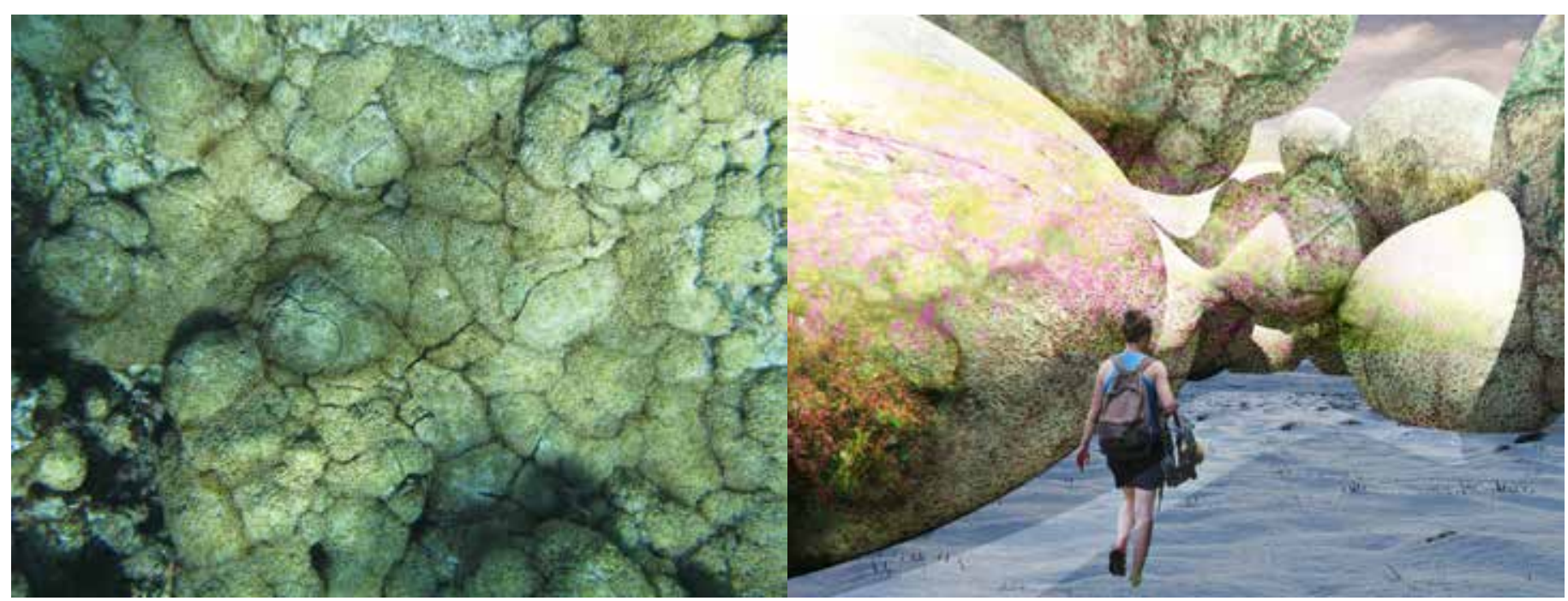

Figure 5. Surface prototypes.
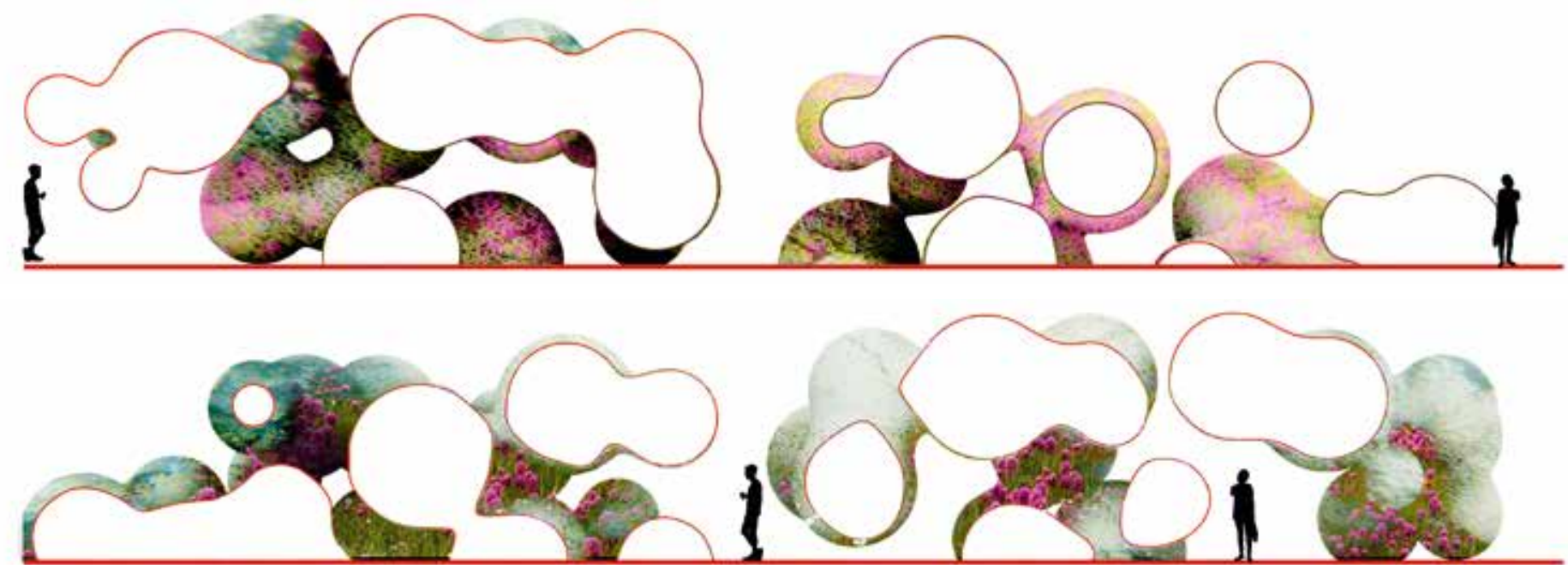

Figure 6. MaterialNature, section 2017. 
mud and clay via wet medium as a slurry that begins to dry within thirty minutes. Once printed and deployed to site, the timeline of each individual module is solely contingent upon the natural processes that take place over the course of the first three years, after which, the individual parts and pieces of the project become a landscape artifact made expressly to be weathered and decayed on site.

As a process, MaterialNature employs a strategy of "opportunistic materiality," building on an attitude of creative problem solving that embodies a forward thinking agenda respondent to the overlooked forces of neglect and waste.

The resultant artifacts of this process theoretically can be any form of material or spatial situation created by discarded materials left behind by humans, but to be more specific for the context of this project, it can build upon the ingredients of the local ecology as building blocks for architectural forms. This agenda positions the future of architecture as a scenario with three possible motives:

1. Potential for relationship with ecology.

2. Potential for relationship with local culture.

3. Potential for relationship with material.

To further contextualize the methodology, this agenda is prescribed to coastal communities. Within this context, 3D printing has been used to replicate the found geologic conditions that assist in erosion control before manmade activities began interfering with the natural processes of beach control. We began taking note of soft rock formations along the shore which
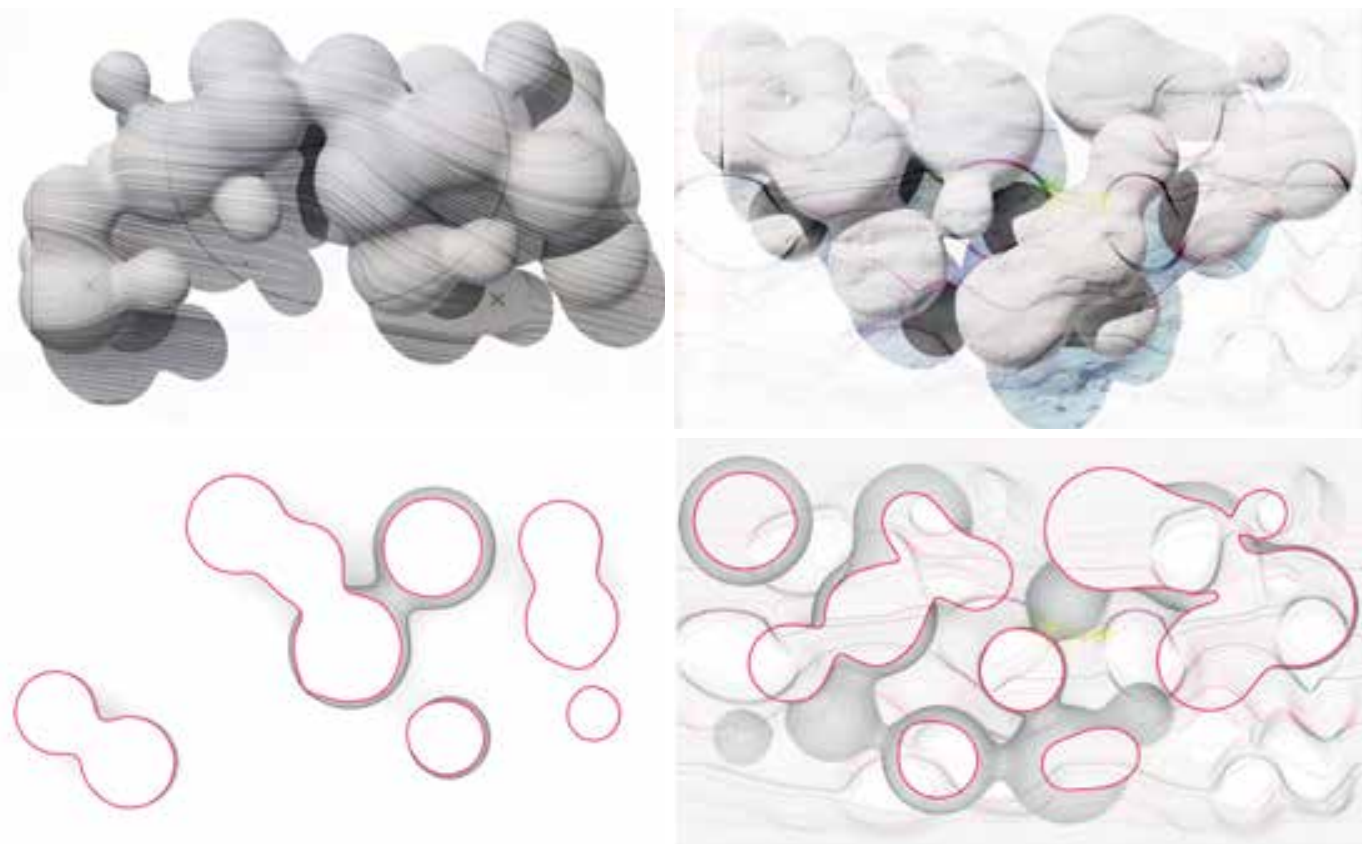

Figure 7. Natural formation prototypes, 2017. 
are transformed by wind, water, and subsequently taken over by plants. The rock here is a porous mixture made of shells and sand which are accepting of spontaneous, opportunistic landscapes. Plants themselves are able to germinate in the rock surfaces and grow year round, creating a surface of landscape on the outlining layer. This is a mutual relationship fostered between the landscape and the local stone which is a concept leveraged by MaterialNature to create a localized architectural surface prototype. In the Architectural Design journal "Experimental Green Strategies, Nature as Measure," the author Terri Peters writes, "nature always doubles up on functions, think of a feather - waterproof, airfoil, self-cleaning, insulating, beauty for sexual reproduction. What can we expect from our buildings." 11 The spirit of programmatic multiplicity and performance is found in MaterialNature as we begin to create surface prototypes that are meant to ride the line between nature as the diviner of form, surface and aesthetic.

The surface prototypes are placed upon circular formations inspired by Moeraki Boulders and Bubbler Crabs. ${ }^{12}$ These boulders are artifacts born from a phenomena that creates spheres of solid rock formed by weather and erosion. Interestingly, many times these types of formations are buried in coastal land without people even knowing about it, with un-natural beach erosion and fracking they appear faster being revealed to the public as an unusual rock formation. These boulders are created by the cementation of mudstone. The main body of the boulders started forming in what was at one time marine mud, near the surface of the Paleocene sea floor. One of the interesting things about these boulders is that some of them

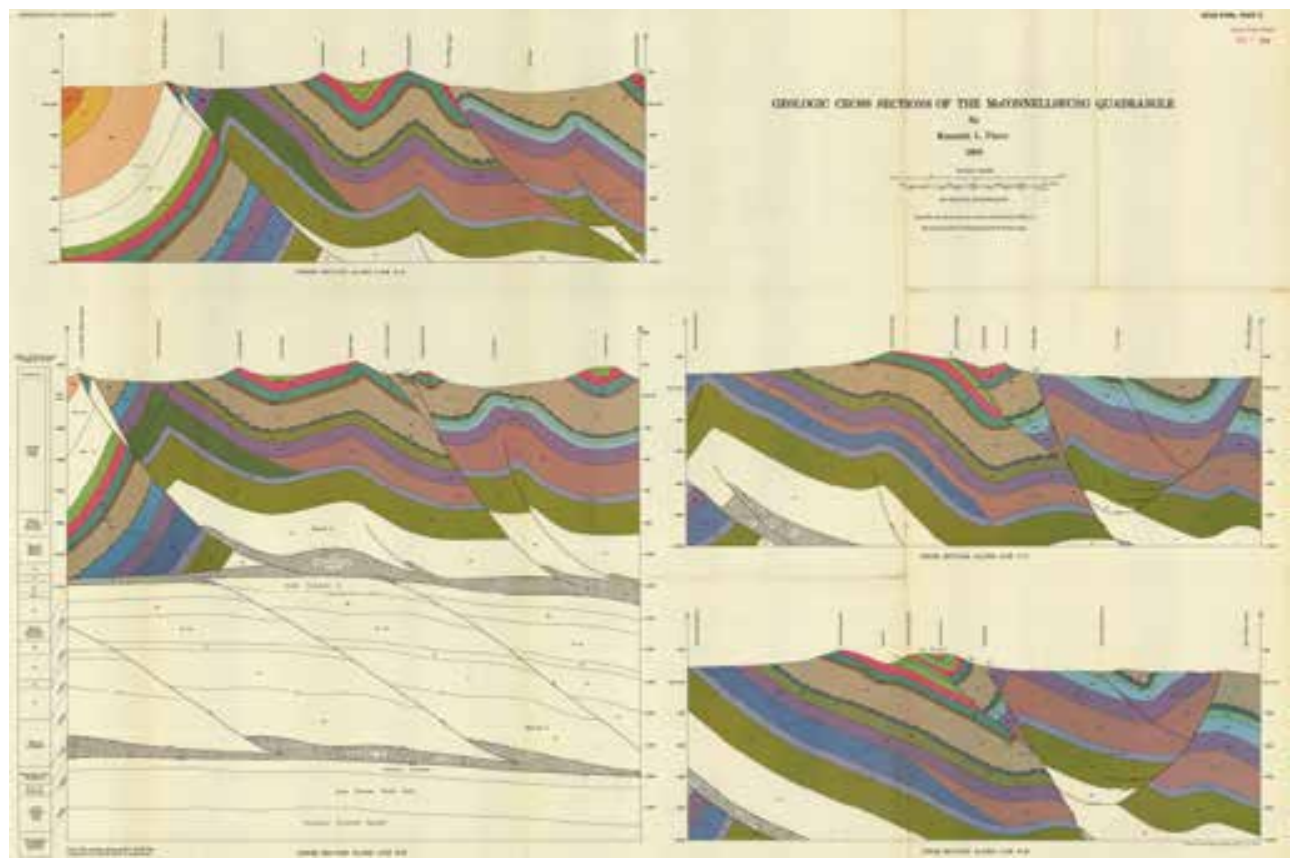

Figure 8. Geologic cross-section, Kenneth Pierce in 1966. 
are hollow on the inside. The boulders consist of mud, fine silt and clay, cemented by calcite. In section, MaterialArtifact begins to take a shape very much inspired by these rocks. The circular forms also make them useful in erosion zones due to the compatibility to drainage and the scalability of each form.

Early geological mappings were used to begin to understand how MaterialNature might have looked as a found condition, as if it were buried based on geological forces and then revealed naturally. To help with calculations, we used Rhinoceros with Grasshopper plug-in Millipede to help alter different data sets affecting the shape grammar. This became an important analysis and optimization tool. It allows for very fast linear elastic analysis of frame and shell elements. In the end, after a significant amount of geologic study and research on rock formation and geography of beaches, MaterialNature took shape as the simplest Millipede distillation of all the forms combining the influences of Moeraki and buried rock. With all of the conceptual underpinnings, it was important that the project be one that indeed took reference from appropriate forces and artifacts, but in the end, it was important that the project become its own entity based on the proscriptive needs of a particular site.

\section{PROJECTIONS}

Though the continued research of geology gave specific shape to the prototypes of MaterialNature, the life span of this architecture is multi-variate, and ultimately fleeting. Its form is meant to change and ultimately become one with its location, perhaps disappearing from its original state. It is quite possible that the self-organizing peripheries will become contaminated once again. However, the project seeks to give credence to nature, allowing it to play a heightened role because of the infrastructure put into place. It is not designed to remain in one state, and is destined to be ephemeral, lasting for only a short, transitory time. It begins as a series of man-made natures and as time progresses, the natural process of beach ecology takes its course; the project develops into a reef like protective landscape, completely natural to the environment. The architecture is a singular transgression between the meta-morphosizing effects of wind currents, geography, landscape germination, pollination, growth and site. Its mission is to embrace integrated site problems in a natural way, with the following problems as being most relevant:

1. Coastal erosion due to man-made activities such as beach grooming, sand removal, drainage and fracking undermine the natural history of place and dilute the natural act of selfmaintenance and performative landscape along the beach.

2. $60 \%$ of U.S. landfills are filled with waste from construction sites or an architecture related industry. The physical act of making 
and designing with traditional materials which are used inefficiently and then thrown out needs to be re-considered due to ecological destruction, new ideas need to be explored, new architectures need to be brought to life that can include ecology and a higher variance of performance.

In many ways, the performance of MaterialNature is contingent upon the placement of the structures. Ideally, they are placed in the wrack zones and drift lines on areas of coastal erosion along shorelines like a land
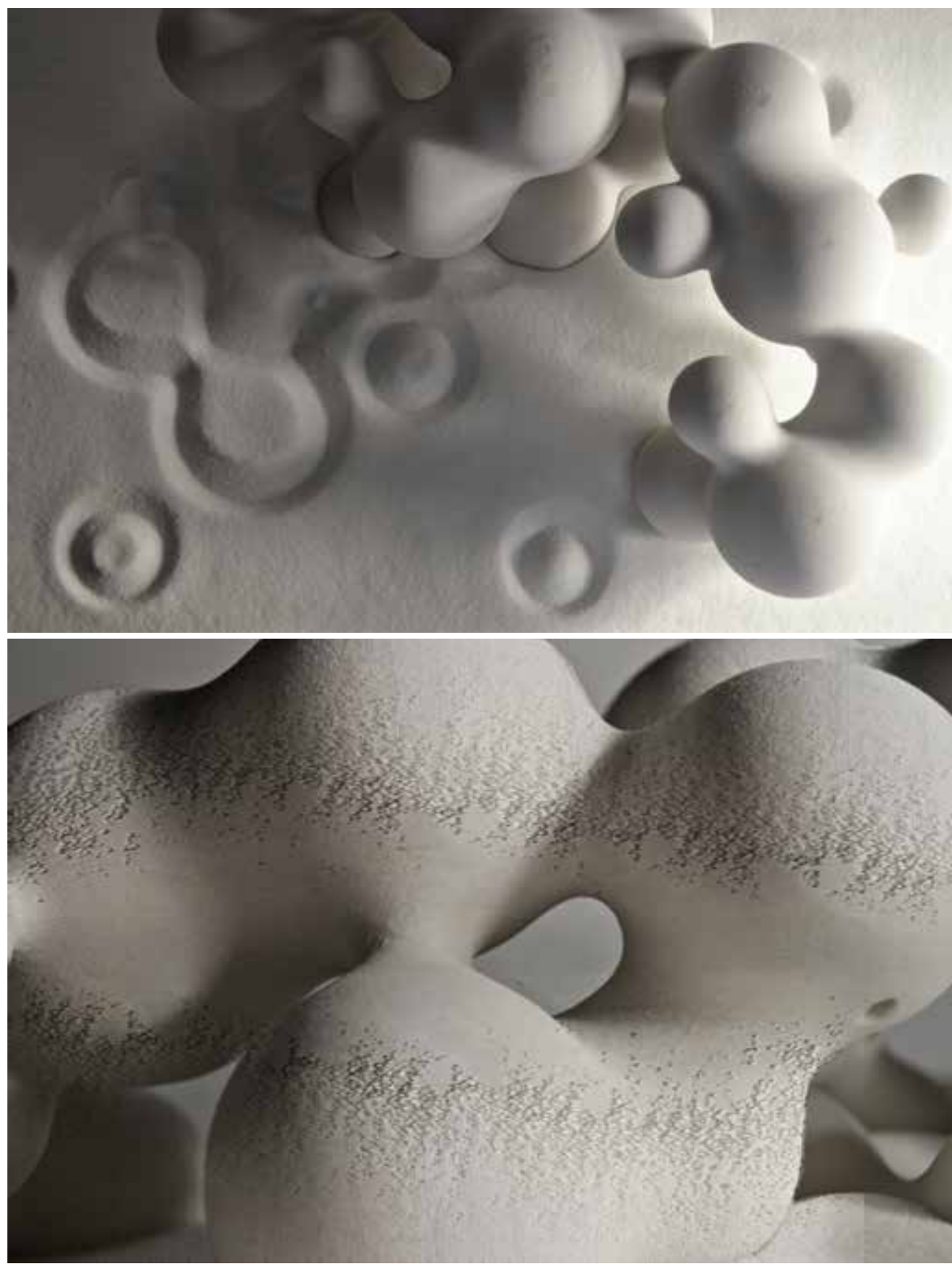

Figure 9. MaterialNature, 3D printed configurations of formations. 
reef. In this situation, the project is introduced as a civic amenity, but also presented as an opportunity for public knowledge and enlightenment by utilizing architecture as a vehicle for issues surrounding local erosion based on recreation, poor land management practices, waste from the ocean going shipping industry, lack of civic design, and the importation of mass produced, seasonal pavilion structures that are non-local and out of touch with a true sense of place. The project leverages large scale 3D printing with sand and clay, and offers an alternative material trajectory paired with the aesthetic of localized ecology. The exterior and structure are all made from the same multi-layered material paste mixture of sand, clay, landscape seeds which are local to the area and responsive to germination but finite enough to pass through our modified printer nozzles. Over time, the architecture becomes garden. The garden becomes landscape. The landscape becomes beach.

\section{Notes}

1. Michael Hensel, "Non-Anthropocentric Architectures," in Performance-Oriented Architecture: Rethinking Architectural Design and the Built Environment, Architectural Design Primers Series (Chichester, UK: John Wiley \& Sons Ltd., 2013).

2. World Commission on Environment and Development, Our Common Future (Oxford, UK: Oxford University Press, 1991)

3. Hensel, "Non-Anthropocentric Architectures."

4. Ibid.

5. Martha Rosler, "In Our Beautiful Future," in "Cities of Dispersal," ed. Rafi Segal and Els Verbakel, special issue, AD Architectural Design 78, no.1 (January 2008), 54-57, http://dx.doi.org/10.1002/ad.601.

6. Orrin H. Pilkey, Tracy Monegan Rice, and William J. Neal, How to Read a North Carolina Beach: Bubble Holes, Barking Sands, and Rippled Runnels (Chapel Hill NC, USA: University of North Carolina Press, 2004.)

7. Clara Cartwright and Rick Wilson, "Beach Ecology - The State of Beach Ecology," Beachapedia, August 2017, http://www.beachapedia.org/Beach_Ecology

8. Jennifer E. Dugan, David M. Hubbard, Henry M. Page, Joshua P. Schimel, "Marine Macrophyte Wrack Inputs and Dissolved Nutrients in Beach Sands," Estuaries and Coasts 34, no.4 (Santa Barbara CA, USA: University of California Press, 2011), 839-850.

9. Jennifer E. Dugan, David M. Hubbard, "Loss of Coastal Strand Habitat in Southern California: The Role of Beach Grooming," Estuaries and Coasts 33, no.1 (Santa Barbara CA, USA: University of California Press, 2010), 67-77.

10. Frei Otto, Biology and Building, Part 1, Institute for Lightweight Structures IL-Series, Vol. 3 (Stuttgart, Ger: University of Stuttgart, 1971).

11. Terri Peters, "Nature As Measure: The Biomimicry Guild," in "Experimental Green Strategies: Redefining Ecological Design," ed. Terri Peters, special issue, AD Architectural Design 81, no.6 (November/December 2011), 44-47, http://dx.doi.org/10.1002/ad.1318. 12. The Moeraki Boulders are a group of very large spherical "stones" on Koekohe Beach near Moeraki on New Zealand's Otago coast. These boulders are actually concretions that have been exposed through shoreline erosion from coastal cliffs that back the beach. "Sand-bubbler crabs, Scopimera and Dotilla (Crustacea, Brachyura, Ocypodidae, Scopimerinae), are small (carapace width $1 \mathrm{~cm}$ ) round-bodied crabs which occur in vast numbers throughout the Indo-Pacific on suitable tropical and sub-tropical sheltered sandy beaches. At high tide they are found in burrows that contain a trapped pocket of air in which the crabs are able to breathe until low tide. During low tide they become active and emerge from their burrows to feed on surface detritus and stranded plankton." David P. Maitland, "Crabs that breathe air with their legs - Scopimera and Dotilla," Nature 319, no. 6053 (1986): 493-495, http://dx.doi.org/10.1038/319493a0. 

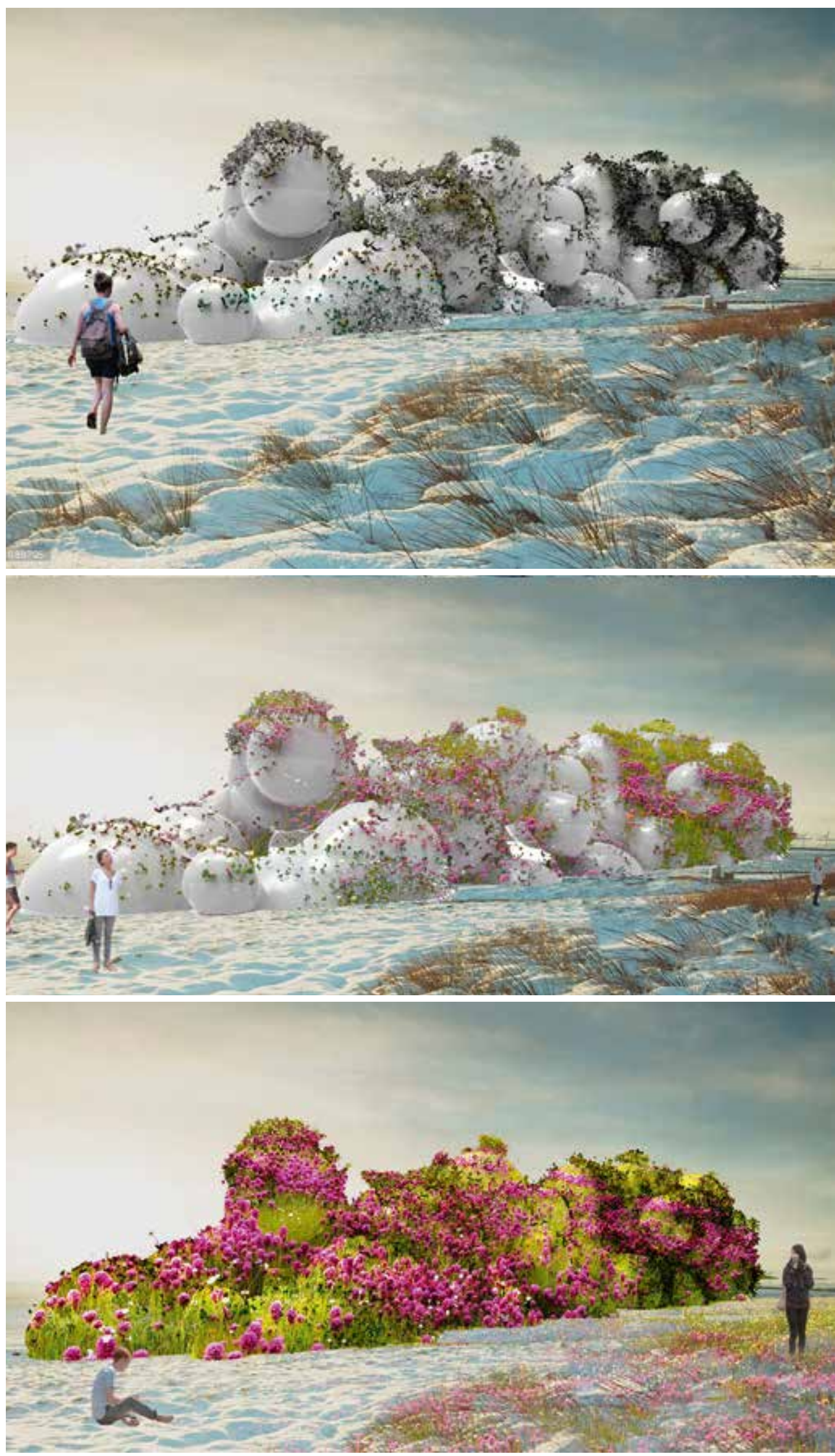

Figure 10. Timeline of MaterialNature moving from year 1 to year 3, 2017. 


\section{Acknowledgments}

This paper is based on a project called MaterialNature originally delivered with the title "Architecture In An Expanded Field, From Interiors To Landscapes" at the 105th ACSA Annual Meeting, "Brooklyn says 'Move to Detroit'," March 23-25, 2017 in Detroit. This project session discussed scholarship and design-based investigations situated at the architectural scale which often slip toward the space of the room or the expanse of the site. Indeed, some of the most often studied projects of recent years operate within this larger field of space making. The topic sought to provide a home for work beyond the precise scale of the building. This essay is a formalized version of the project talk given at this event in which MaterialNature was in schematic design phase. While still in development, this project has benefitted from being delivered orally and now formalized as an essay. It will continue to develop as an architectural logic and in many ways is still a conceptual portrait of an architecture that will ultimately act as an approach to the design of ecologically dominant structures.

\section{Credits}

Figure 1: photo by John Batchelder, public domain.

Figures 2-10: images and photos are an elaboration of the Author.

Marcus Farr is the studio leader of Material Artifact. He is an Assistant Professor of Architecture at the American University of Sharjah in the United Arab Emirates. He studied architecture at Rice University and Drury University. He has practice experiences throughout the United States, Europe, Middle East and Asia. He has published on, amongst others: Landscape Architecture Magazine, Texas Architect, Architectural Record, Architect, Cite Magazine, The New York Times, and World Architecture Magazine. His teaching focuses on design methods and materials technology. His research focuses on sustainability through opportunistic and vernacular material strategies. E-mail: marcusfarr@mac.com 